\title{
Biological synthesis and characterization of intracellular gold nanoparticles using biomass of Aspergillus fumigatus
}

\author{
PRANAV VASANTHI BATHRINARAYANAN, DILLIGANESH THANGAVELU, VASANTH KUMAR \\ MUTHUKUMARASAMY, CHAMUNDEESWARI MUNUSAMY and BASKAR GURUNATHAN* \\ Department of Biotechnology, St. Joseph's College of Engineering, Chennai 600 119, India
}

MS received 18 July 2012; revised 1 September 2012

\begin{abstract}
Nanotechnology is emerging as one of the most important and revolutionizing area in research field. Nanoparticles are produced by various methods like physical, chemical, mechanical and biological. Biological methods of reduction of metal ions using plants or microorganisms are often preferred because they are clean, nontoxic, safe, biocompatible and environmentally acceptable. In the present study, Aspergillus fumigatus was used for the intracellular synthesis of gold nanoparticles. Stable nanoparticles were produced when an aqueous solution of chloroauric acid $\left(\mathrm{HAuCl}_{4}\right)$ was reduced by $\mathrm{A}$. fumigatus biomass as the reducing agent. Production of nanoparticles was confirmed by the colour change from yellow to pinkish violet after $\sim 72 \mathrm{~h}$ of reaction. The produced nanoparticles were then characterized by Fourier transform infrared spectroscopy (FT-IR), scanning electron microscope (SEM), energy dispersive spectroscopy (EDS) and X-ray diffraction spectroscopy (XRD). SEM images of sample revealed that the nanoparticles were spherical, irregularly shaped with indefinite morphology. Biosynthesized gold nanoparticles were in the range of 85.1-210 $\mathrm{nm}$ in size. The presence of gold nanoparticle was confirmed by EDS analysis. Crystalline nature and face-centred cubic structure of synthesized gold nanoparticle was confirmed by XRD pattern.
\end{abstract}

Keywords. Gold nanoparticles; biosynthesis; fungal biomass; characterization; energy dispersive spectroscopy.

\section{Introduction}

Nanotechnology mainly focuses on the development of synthetic as well as natural systems for the production of structures and materials at nanoscale (Absar et al 2005). In recent years, metal nanoparticles have been the subject of interest due to their unique physical, chemical and optical properties (Ahmad et al 2007). These unique properties arise due to their small size and large specific surface area. Hence, the metal nanoparticles have a wide range of applications from electronics to medicine and from catalysis to photonics. (Avinash et al 2009). Nanoparticles can be synthesized from physical and chemical methods. The simplest method for the production of nanoparticles is the reduction of their respective salts (Rashmi and Preeti 2009). Other strategies include lithography, sonochemical processing, cavitation processing, micro-emulsion processing, UV irradiation and high energy ball-milling. However, these methods are expensive, toxic and involve the use of harmful chemicals apart from other complexities like low stability of the produced nanoparticles and aggregation of the particles (Raffi 2007).

Hence, in order to produce the nanoparticles by clean, non-toxic, safe, biocompatible and environmentally acceptable methods, many biological systems have been used to

*Author for correspondence (basg2004@gmail.com) produce the nanoparticles both intracellularly and extracellularly (Boisselier and Astruc 2009). Some well known examples include the use of bacteria, fungi and plants for the production of nanoparticles. Accumulation of metal ions by microbes has been regarded as low-cost, eco-friendly and easily achievable (Chan 2006). Especially fungi are often used in the production of metal nanoparticles. Since fungi have several advantages over bacteria, they are often preferred. Some of the advantages of fungal sources for the production of metal nanoparticles include high tolerance towards metals, high wall-binding capacity, can be easily scaled up, easy to culture on a large scale and ability to secrete large amount of enzymes (Zeinab et al 2011). Kuber and Dsouza (2006) reported the use of fungus Aspergillus fumigatus for the extracellular biosynthesis of silver nanoparticles. Zahra et al (2010) also reported the same fungus Aspergillus fumigatus for the production of silver nanoparticles, thus proving that this fungus is an excellent candidate for the biosynthesis of nanoparticles. Aspergillus terreus, on the other hand, has been used by Rashmi and Preeti (2009) for the biomimetic synthesis and characterization of protein capped silver nanoparticles.

Greener synthesis of nano gold-biocomposite by fungus Cylindrocladium floridanum was reported by Kannan and Natarajan (2011a). C. floridanum accumulated gold nanoparticles on the surface of the mycelia, when it was cultured in static condition for a period of 7 days. Thoomatti and Peramchi (2011) used Fusarium oxysporum for both 
intracellular and extracellular production of gold nanoparticles. The rapid reduction of metal ions resulting in the formation of stable silver and gold nanoparticles of variable size and shape were reported. Greener synthesis of anisotrophic nanostructures and isotrophic spherical gold nanoparticles using the cell-free filtrate of fungus Sclerotium rolfsii was reported by Kannan and Natarajan (2011b). It was reported that NADPH-dependent enzyme present in the cell-free filtrate of $S$. rolfsii, when incubated with the aqueous chloroauric acid solution has the ability to synthesize gold nanoparticles at ambient temperature. The biosynthesis of gold nanoparticles by the fungus Epicoccum nigrum isolated from Andalian gold mine in north-west of Iran was reported by Zeinab et al (2011). The gold nanoparticles were produced both intra and extracellularly by reaction of an aqueous solution of chloroauric acid with the biomass of fungus E. nigrum. However, there was no report on the production of gold nanoparticles using A. fumigatus. Hence, the present work was focused on the production of intracellular gold nanoparticles using $A$. fumigatus.

\section{Materials and methods}

\subsection{Fungi growth conditions}

The fungus Aspergillus fumigatus was obtained from IMTECH, Microbial Type Culture Collection (MTCC), Chandigarh, India. The fungus was subcultured by growing on Czepak agar slants for $72 \mathrm{~h}$. The temperature was maintained at $30^{\circ} \mathrm{C}$.

\subsection{Biosynthesis of gold nanoparticles using fungus Aspergillus fumigatus}

The fungus A. fumigatus was grown aerobically in $500 \mathrm{~mL}$ Erlenmeyer flask containing $200 \mathrm{~mL}$ of liquid growth medium (potassium dihydrogen phosphate, yeast extract, sucrose, sodium nitrate, ferrous sulphate, magnesium sulphate, potassium chloride and distilled water). The culture was agitated in an orbital shaker at $160 \mathrm{rpm}$ in the temperature range of $32{ }^{\circ} \mathrm{C}$ for $72 \mathrm{~h}$. After 3 days, the biomass was separated from the culture broth by filtration using a suction pump and a Wattman paper. The biomass was repeatedly washed with distilled water to remove medium components. $5 \mathrm{~g}$ of biomass was suspended in $200 \mathrm{~mL}$ of $0.001 \mathrm{M}$ aqueous chloroauric acid at pH 2.5 in $500 \mathrm{~mL}$ Erlenmeyer flask. The flask was agitated at $160 \mathrm{rpm}$ for $72 \mathrm{~h}$ at $32^{\circ} \mathrm{C}$. The entire process was carried out in complete darkness.

\subsection{Characterization of gold nanoparticles}

The nature of the gold nanoparticles was studied using XRD analysis. The samples for XRD were prepared by collecting the fungal biomass after centrifuging at $8000 \mathrm{rpm}$ for $10 \mathrm{~min}$, drying the fungal biomass in hot air oven at $60^{\circ} \mathrm{C}$ overnight.
$1 \mathrm{~g}$ of the powdered sample was taken and XRD analysis was performed. Fungal biomass before and after the formation of gold nanoparticles was examined by SEM on a QUANTA 200 equipped with energy dispersive spectroscopy (EDS). EDS analysis is done to get an indication of the amount of gold nanoparticles present in the biomass. The samples were prepared in the same way as described for XRD and SEM analyses and $500 \mathrm{mg}$ of the sample was analysed. The samples were then mixed with a small amount of $\mathrm{KBr}$ (binding agent) using a clean mortar and a pestle. The mixture was then punched into pellets using a hydraulic press. The pellets were then subjected to FT-IR analysis on a BRUKER $\alpha$-T FT-IR spectrometer.

\section{Results and discussion}

\subsection{Visual observation}

Gold nanoparticles were produced using fungus A. fumigatus by the method described in chapter 2.2. On mixing the fungal biomass with the aqueous solution of chloroauric acid, the colour of the biomass was changed from yellow to purple. The colour change indicated the reduction of the chloroauric acid ions by the fungal enzyme, which resulted in the formation of gold nanoparticles.

\subsection{Structural characterization of gold nanoparticles using SEM}

Structural features of the produced gold nanoparticles were characterized using SEM. As the metal particles are good conductors, they can be observed without any prior carbon coating at a magnification of $1000 \times$ in a voltage of $10 \mathrm{kV}$. Figures 1(a) and (b) are SEM images of A. fumigatus biomass after the addition of the chloroauric acid at $2 \mu \mathrm{m}$ and $500 \mathrm{~nm}$, respectively. It was identified from SEM images that the fungal mycelia was loaded with glittering particle. This depicts that the glittering particles on the mycelia should be gold nanoparticles accumulated on the mycelia intracellularly. Gold nanoparticles loaded in the mycelia were found to be in the size range of $85 \cdot 1-210 \mathrm{~nm}$.

\section{$3.3 \quad$ FT-IR spectrum analysis}

The synthesized gold nanoparticles were subjected to FTIR analysis to find out the bioactive compounds synthesized by the fungus and associated with the nanoparticles. FT-IR images of A. fumigatus samples show a number of functional bonds associated with them which provide them with stability by capping them. From figure 2, it can be inferred that samples have peaks in the range of 1634, 2078, 3345 and $3600 \mathrm{~cm}^{-1}$. 1634 corresponds to $\mathrm{C}=\mathrm{O}$ bond, $2078 \mathrm{co}-$ rresponds to $\mathrm{C}-\mathrm{N}$ bond, 3345 corresponds to $\mathrm{N}-\mathrm{H}$ bond and 3600 corresponds to $\mathrm{O}-\mathrm{H}$ bond. 

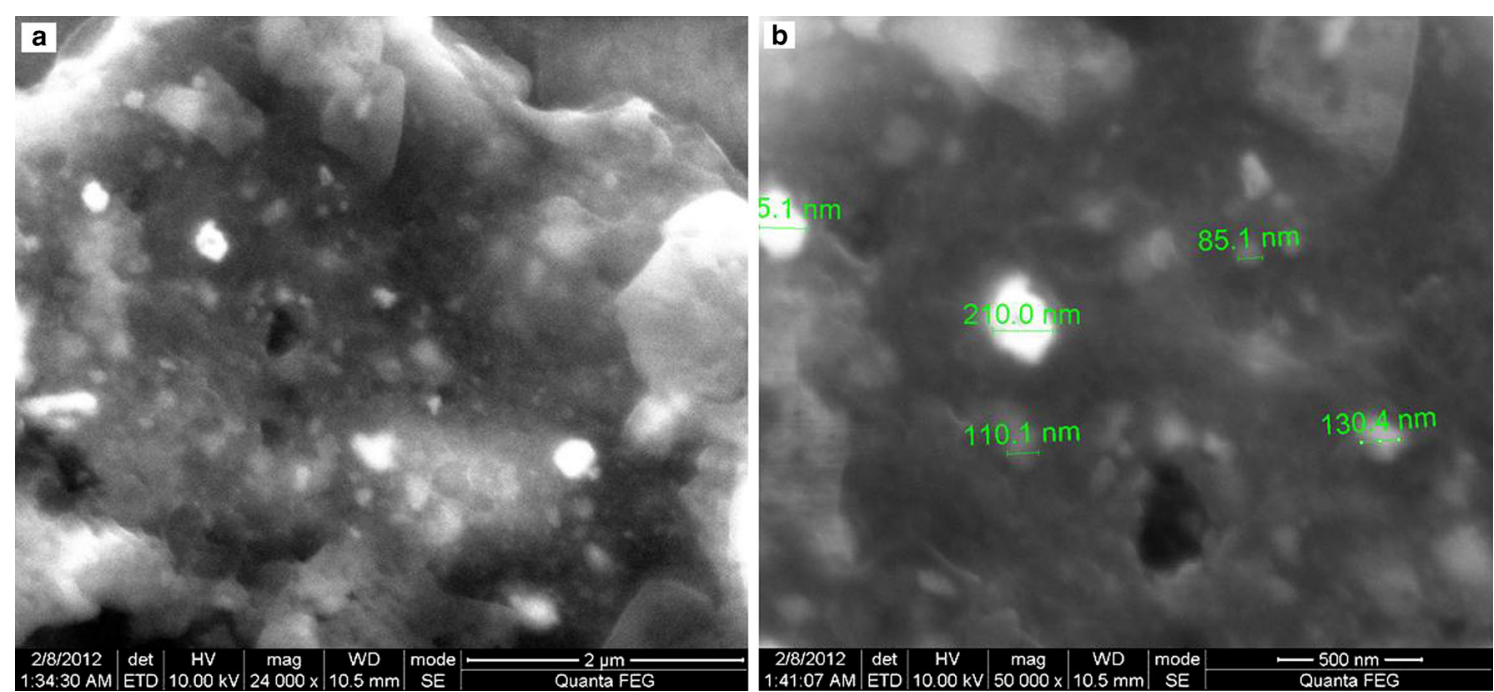

Figure 1. SEM image of A. fumigatus: (a) showing presence of glittering gold nanoparticles on mycelia at $2 \mu \mathrm{m}$ and (b) showing size range of the gold nanoparticles at $500 \mathrm{~nm}$.

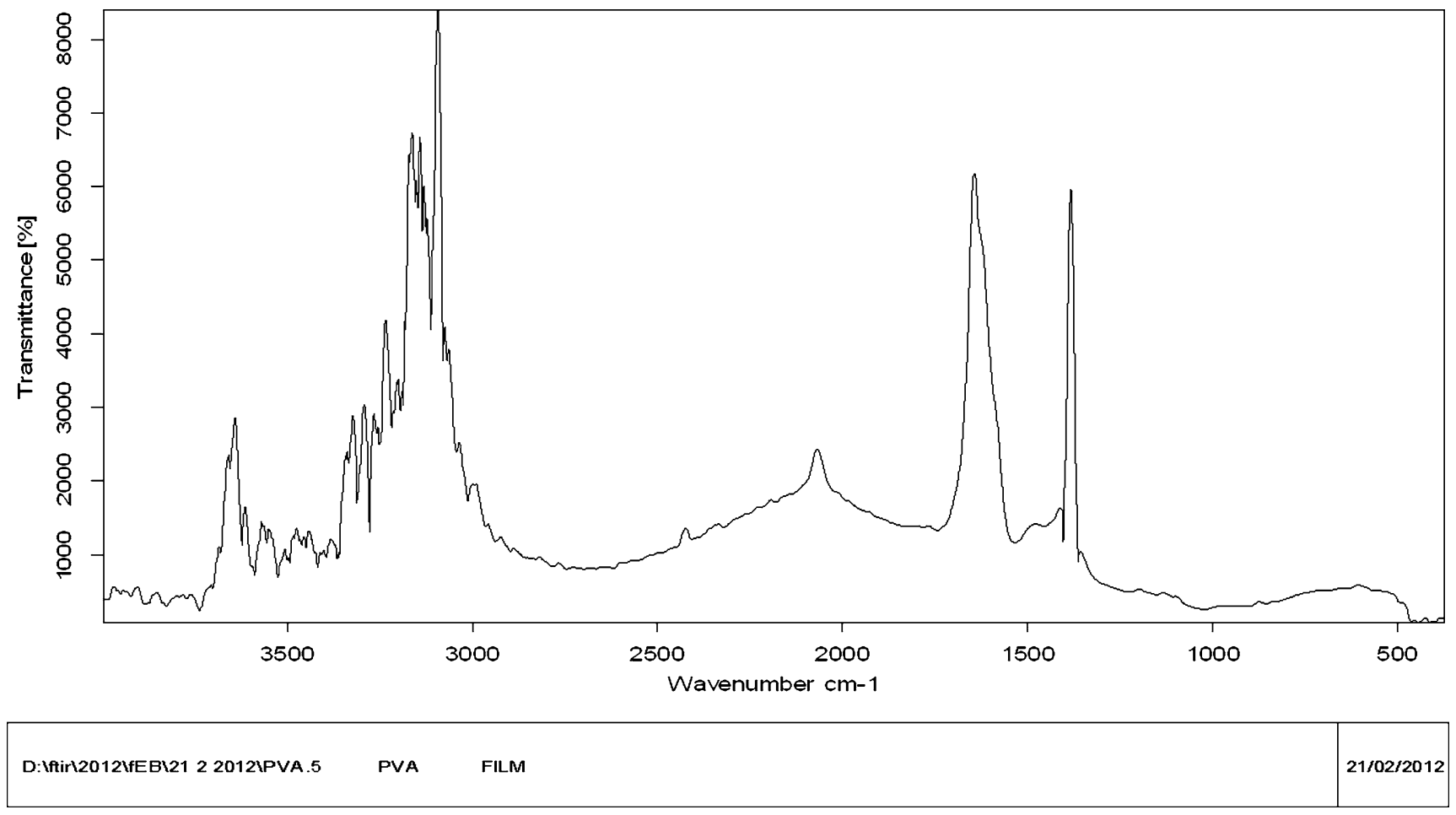

Figure 2. Smooth FT-IR spectrum for A. fumigatus showing peaks at different places. Each of these peaks are associated with specific bonds.

\subsection{XRD analysis}

XRD image of the sample after the addition of gold chloride hydrate is depicted in figure 3. A strong signal can be seen at 31.9 which might have arised from some biomass or media components. Figure 3 represents XRD pattern of the produced gold nanoparticles. Peak position at 37.8 in red peak pattern represents the presence of gold and the value is consistent. Although figure 3 is in agreement with Bragg's reflection values at $2 \Theta$, the produced gold nanoparticles exibit irregular morphology. XRD patterns clearly show that both the nanoparticles are crystalline in nature. 


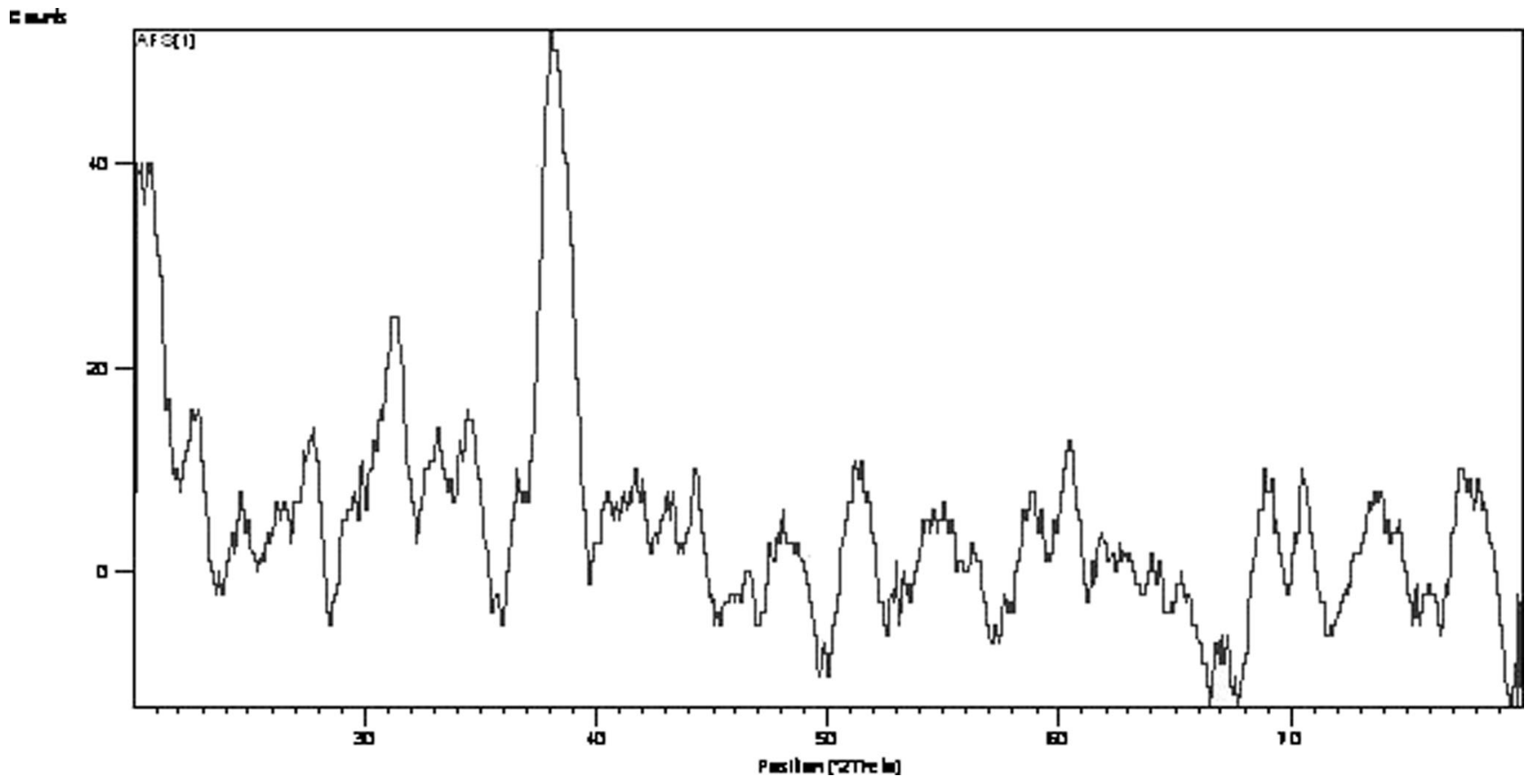

Figure 3. XRD pattern for A. fumigatus shows peak at 37.8, which is in agreement with standard peak value of gold nanoparticles. Bragg's reflections were also identified.

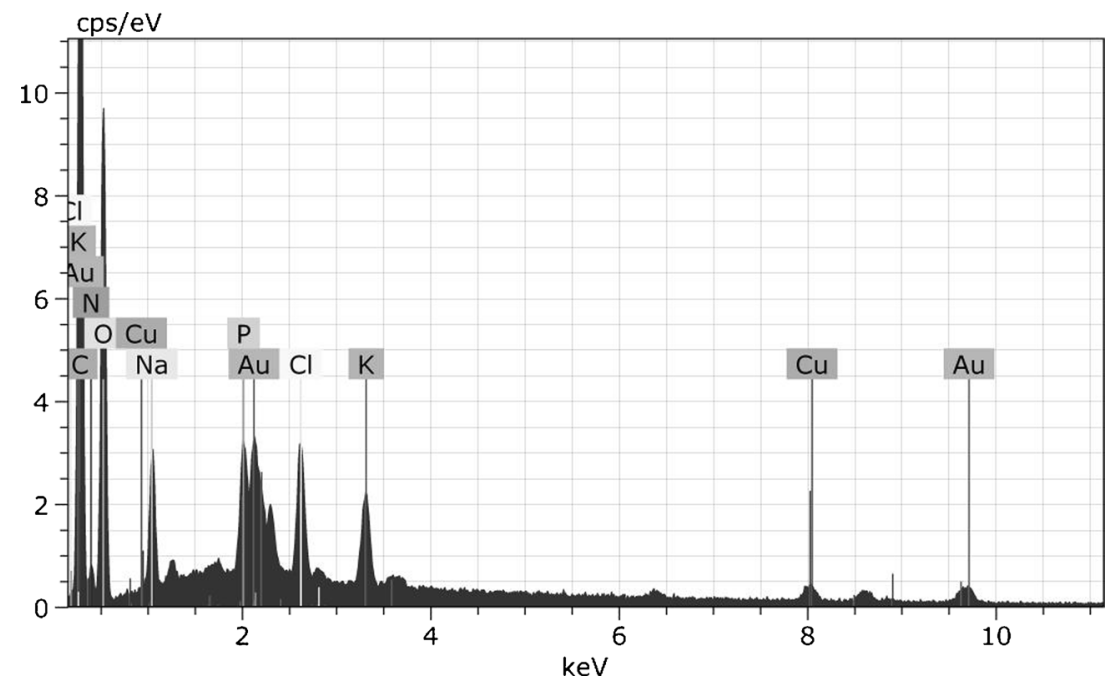

Figure 4. EDS pattern for A. fumigatus showing strong signals for gold nanoparticles at three different places. Signals of other chemicals might have arised due to media components or during growth of fungi.

\subsection{EDS analysis}

The energy dispersive spectroscopic analysis is done to get an indication of the amount of gold nanoparticles present in the biomass. EDS analysis of thin film of fungal biomass shows strong signals for gold atoms along with weak signals from oxygen and potassium. These weak signals could have arisen from macromolecules like proteins/enzymes and salts of fungal biomass. Figure 4 shows presence of gold which is the gold nanoparticles.

\section{Conclusions}

Gold nanoparticles produced by Aspergillus fumigatus were in the size range of $85 \cdot 1-210 \mathrm{~nm}$ and found to be spherical in shape and had irregular morphologies which were confirmed by SEM analysis. The presence of the gold nanoparticles was confirmed by EDS analysis. The presence of functional groups was confirmed by FT-IR analysis with the peaks in the range of $350-3650 \mathrm{~cm}^{-1}$. The different functional groups associated were found to be $\mathrm{C}=\mathrm{O}, \mathrm{C}-\mathrm{N}, \mathrm{N}-\mathrm{H}$, 
$\mathrm{O}-\mathrm{H}$. These bonds were found to provide stability to the produced nanoparticles by capping them. XRD results showed that Bragg's reflection was in accordance with gold nanoparticles with $2 \theta=37 \cdot 8$. Thus, A. fumigatus was found to be a good candidate for the production of gold nanoparticles.

\section{Acknowledgments}

The authors would like to thank SRM University, Chennai for their SEM-EDS, XRD and FT-IR analyses.

\section{References}

Absar A, Satyajyoti S, Khan M I, Rajiv K and Sastry M 2005 J. Biomed. Nanotechnnol. 147
Ahmad R S, Sara M, Shahverdi H M, Hossein J and Ashraf A N 2007 Process Biochem. 42919

Avinash I, Mahendra R, Aniket G and Manisha B 2009 J. Nanopart. Res. 112079

Boisselier E and Astruc D 2009 Chem. Soc. Rev. 381759

Chan W C W 2006 Biol. Blood Marrow Transplant. 1287

Kannan B and Natarajan S 2011a J. Hazard Mater. 189519

Kannan B and Natarajan S 2011b Colloid Surf. A 380156

Kuber C B and Dsouza S F 2006 Colloids Surf. B 47160

Raffi M 2007 PhD thesis, Pakistan Institute of Engineering \& Applied Sciences (Islamabad: Pakistan research repository)

Rashmi S and Preeti V 2009 Bioresour. Technol. 100501

Thoomatti S A and Peramchi P 2011 Dig. J. Nanomater. Bios. 6 1587

Zahra R N, Mohammad P and Farah S H 2010 Iran J. Biotechnol. 8 56

Zeinab S, Mojtaba S and Farzad K 2011 J. Clust. Sci. 22661 